\title{
FEED-DOWN EFFECT IN DIPOLE ALIGNMENT
}

\author{
A.Verdier, E.Wildner, CERN, Geneva, Switzerland
}

\section{Abstract}

The field of strong super conducting magnets contains substantial multipole errors. An upper limit of their value is specified on the reference closed orbit. When the dipole is installed in a real machine, its axis cannot be exactly on the reference orbit, and "feed-down multipoles" are introduced. This paper describes an analytical approach to the alignment specification associated with this effect with an example from the LHC at CERN.

\section{THE LHC DIPOLE}

The LHC Dipole has unavoidable field shape imperfections. For some components of these field imperfections there is a spool piece corrector mounted at the end of the dipole to compensate the average value of the field error component. Every dipole has a sextupolar corrector and every second dipole has a combined octupolar/decapolar corrector. The usual expansion of the dipole field reads if we assume a two dimensional field along the dipole axis, $\mathrm{x}$ being the horizontal and $\mathrm{y}$ the vertical co-ordinate:

$$
B_{y}+i B_{x}=B_{r e f} \sum_{n=1}^{\infty}\left(b_{n}+i a_{n}\right)\left(\frac{x+i y}{R_{r e f}}\right)^{n-1}
$$

where, in European convention, $\mathrm{n}=1$ identifies a dipole field $n=2$ a quadrupole etc. The $a$ and the $b$ indicate the skew and normal components of the field respectively, $R_{\text {ref }}$ is the reference radius (17mm) for the LHC. The feed down harmonics due to $\left(a_{n}, b_{n}\right)$ harmonics, can be calculated by changing $\mathrm{x}$ and $\mathrm{y}$ in the formula (1) into $\mathrm{x}_{0}+\mathrm{x}$ and $\mathrm{y}_{0}+\mathrm{y}$ respectively. Then the relevant monomials associated with a given order $\mathrm{k}$ are kept. We obtain:

$b_{k}+i a_{k}=\left(b_{n}+i a_{n}\right)\left[\frac{(n-1) !}{(k-1) !(n-k) !}\right]\left(\frac{x_{0}+i y_{0}}{R_{r e f}}\right)^{n-k}(2)$ where $\mathrm{k}$ is the order of the feed-down multipole, with $\mathrm{k}<\mathrm{n}$.

Figure 1 illustrates the case when there is only one corrector $\left(b_{3}\right)$. The dipole centre is displaced relative to the reference closed orbit by $\mathrm{dz}$ where the $\mathrm{z}$-coordinate represents either the horizontal or the vertical displacement. $\mathrm{dz}_{\mathrm{s}}$ is the misalignment of the corrector relative to the average dipole axis. $\mathrm{dz}_{\mathrm{s}}$ has one contribution from the displacement of the corrector relative to the dipole axis and one contribution that originates from the pitch (yaw) of the dipole. For this configuration $\mathrm{dz}$ induces feed-down for components that are not compensated. For the compensated component the corrector misalignment (dz-dzs) contributes with $-\mathrm{b}_{3}$ and the dipole misalignment contributes with $b_{3}$.

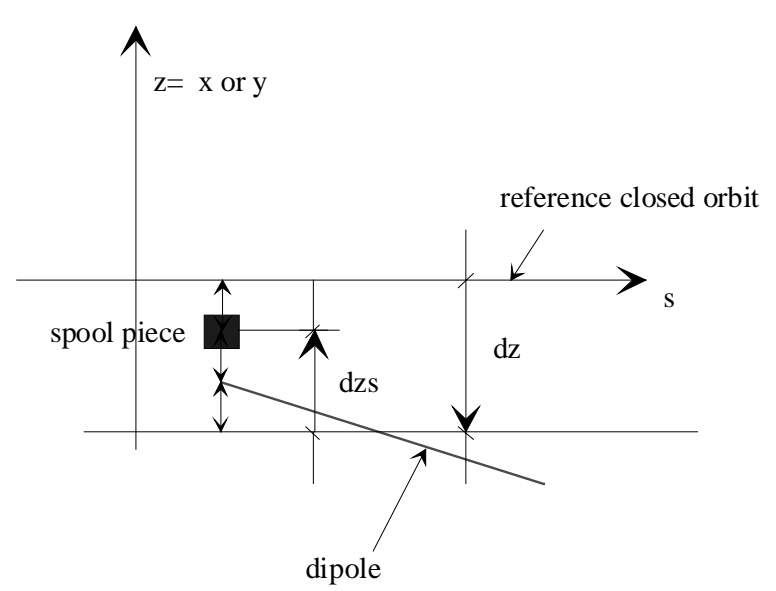

Figure 1: Configuration for a dipole with a field error corrector. $\mathrm{dz}_{\mathrm{s}}$ is the displacement of the corrector relative to the average axis of the dipole, $\mathrm{dz}$ is the average displacement of the dipole.

For the case where every second dipole has a corrector, the corrector misalignment (dz1-dzs) contributes with $2 b_{4}$ or $-2 b_{5}$ and the dipole misalignment $\mathrm{dz} 1$ or $\mathrm{dz} 2$ contributes with $b_{4}$ or $b_{5}$. For the not compensated components the contribution comes from dz1 and dz2 for dipole 1 and dipole 2 respectively. The correctors have a random contribution of 2 times their value.

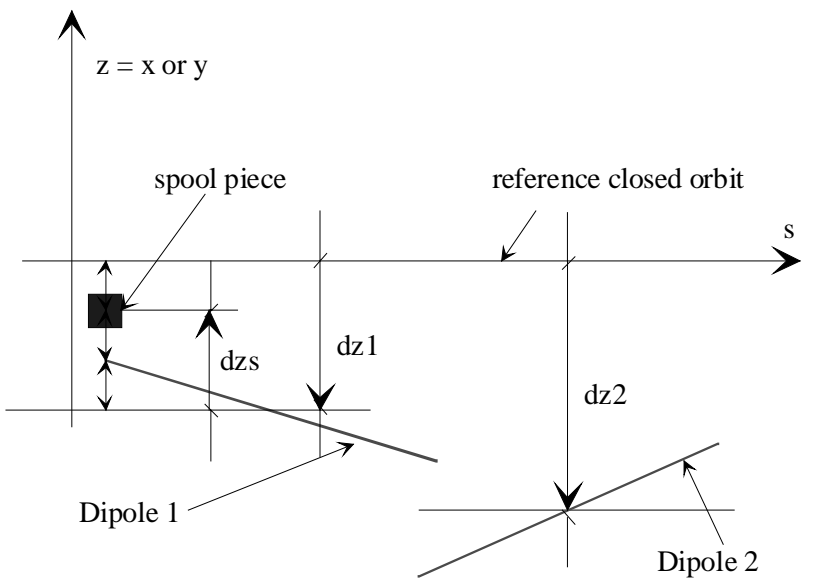

Figure 2: Configuration where one dipole out of two has a corrector. 


\section{DERIVATION OF EXPRESSIONS FOR ALIGNMENT TOLERANCES}

From formula (2) follows with $\mathrm{n}-\mathrm{k}=1$, first order feeddown, dipole without corrector

$$
\begin{aligned}
& -\left|b_{t o l}\right| \leq(n-1) b_{n} \frac{d x}{R_{r e f}}-(n-1) a_{n} \frac{d y}{R_{r e f}} \leq\left|b_{t o l}\right| \\
& -\left|a_{t o l}\right| \leq(n-1) a_{n} \frac{d x}{R_{r e f}}+(n-1) b_{n} \frac{d y}{R_{r e f}} \leq\left|a_{t o l}\right|
\end{aligned}
$$

where the suffix tol means tolerance. Solving this sytem gives

$$
\begin{aligned}
& |d x|<\frac{\left|b_{n} b_{t o l}\right|+\left|a_{n} a_{t o l}\right|}{\left(b_{n}^{2}+a_{n}{ }^{2}\right)} \frac{R_{r e f}}{(n-1)} \\
& |d y|<\frac{\left|a_{n} b_{t o l}\right|+\left|b_{n} a_{t o l}\right|}{\left(b_{n}{ }^{2}+a_{n}{ }^{2}\right)} \frac{R_{r e f}}{(n-1)}
\end{aligned}
$$

For the dipoles with corrector, the contribution from the misalignment of the dipole is $\mathrm{dx}_{\mathrm{d}}$ and the contribution from the corrector is $\left(\mathrm{dx}_{\mathrm{s}}+\mathrm{dx}_{\mathrm{d}}\right)$ relative to the reference orbit (similarly in the direction of the $y$-co-ordinate)

$$
\begin{aligned}
& b_{t o l, d}+i a_{t o l, d}=\left(b_{n}+i a_{n}\right)\left(d x_{d}+i d y_{d}\right) \frac{(n-1)}{\left(R_{r e f}\right)} \\
& b_{t o l, s}+i a_{t o l, s}=\left(-b_{n}\right)\left(\left(d x_{s}+d x_{d}\right)+i\left(d y_{s}+d y_{d}\right)\right) \frac{(n-1)}{\left(R_{r e f}\right)}
\end{aligned}
$$

this gives the following, which can be solved only with an assumption about the relation between $\mathrm{dx}_{\mathrm{d}}$ and $\mathrm{dx}_{\mathrm{s}}$, which is set to $\alpha^{*} \mathrm{dx}_{\mathrm{d}}$ (the corrector tolerance is a fraction of the dipole tolerance), similarly for the $\mathrm{y}$-direction, $\mathrm{dy}_{\mathrm{s}}$ is set to $\beta * \mathrm{dy}_{\mathrm{d}}$.

$$
\begin{aligned}
& -\left|b_{t o l}\right| \leq-(n-1) b_{n} \frac{d x_{s}}{R_{r e f}}-(n-1) a_{n} \frac{d y_{d}}{R_{r e f}} \leq\left|b_{t o l}\right| \\
& -\left|a_{t o l}\right| \leq(n-1) a_{n} \frac{d x_{d}}{R_{r e f}}-(n-1) b_{n} \frac{d y_{s}}{R_{r e f}} \leq\left|a_{t o l}\right|
\end{aligned}
$$

Solving for $\mathrm{dx}_{\mathrm{d}}$ and $\mathrm{dy}_{\mathrm{d}}$

$$
\begin{aligned}
& \left|d x_{d}\right|<\frac{\left|\beta b_{n} b_{t o l}\right|+\left|a_{n} a_{t o l}\right|}{\left(\alpha \beta b_{n}{ }^{2}+a_{n}{ }^{2}\right)} \frac{R_{r e f}}{(n-1)} \\
& \left|d y_{d}\right|<\frac{\left|a_{n} b_{t o l}\right|+\left|\alpha b_{n} a_{t o l}\right|}{\left(\alpha \beta b_{n}{ }^{2}+a_{n}{ }^{2}\right)} \frac{R_{r e f}}{(n-1)}
\end{aligned}
$$

This is valid for systematic components and is the same for dipole with one or two correctors.

For the random effect we have to distinguish the dipole with and without corrector for the components b4 and b5. Starting it over again, now scaling $x$ and y by $R /(n-1)$, for simpler expressions

$$
b_{t o l}+i a_{t o l}=(x+i y)\left(b_{n}+i a_{n}\right)
$$

$$
\begin{aligned}
& b_{t o l}=x b_{n}-y a_{n} \\
& a_{t o l}=y b_{n}+x a_{n}
\end{aligned}
$$

With $\mathrm{x}_{\mathrm{d} 1}$ and $\mathrm{x}_{\mathrm{d} 2}$ taken as the distance from the dipole 1 and 2 respectively from the closed orbit and $x_{s, p}$ as the distance of the spool piece from the dipole

$$
\begin{gathered}
b_{t o l}=b_{n}\left(x_{d 1}+x_{d 2}\right)-a_{n}\left(y_{d 1}+y_{d 2}\right)-2 b_{n}\left(x_{d 1}+x_{s}\right) \\
a_{t o l}=b_{n}\left(y_{d 1}+y_{d 2}\right)+a_{n}\left(x_{d 1}+x_{d 2}\right)-2 b_{n}\left(y_{d 1}+y_{s}\right) \\
b_{t o l}=b_{n}\left(x_{d 2}-x_{d 1}\right)-a_{n}\left(y_{d 1}+y_{d 2}\right)-2 b_{n} x_{s} \\
a_{t o l}=b_{n}\left(y_{d 2}-y_{d 1}\right)+a_{n}\left(x_{d 1}+x_{d 2}\right)-2 b_{n} y_{s}
\end{gathered}
$$

If we assume the expectation values, $b_{n}, a_{n}, x 1, x 2, y 1, y 2$ uncorrelated we get

$$
\begin{aligned}
b_{t o l}{ }^{2}= & b_{n}{ }^{2}\left(x_{d 2}{ }^{2}+x_{d 1}{ }^{2}\right)+a_{n}{ }^{2}\left(y_{d 1}{ }^{2}+y_{d 2}{ }^{2}\right)+4 b_{n}{ }^{2} x_{s}{ }^{2} \\
a_{t o l}{ }^{2}= & b_{n}{ }^{2}\left(y_{d 1}{ }^{2}+y_{d 2}{ }^{2}\right)+a_{n}{ }^{2}\left(x_{d 1}{ }^{2}+x_{d 2}{ }^{2}\right)+4 b_{n}{ }^{2} y_{s}{ }^{2} \\
& b_{t o l}{ }^{2}>2 b_{n}{ }^{2} \sigma_{x d}{ }^{2}+2 a_{n}{ }^{2} \sigma_{y d}{ }^{2}+4 b_{n}{ }^{2} \sigma_{x s}{ }^{2} \\
& a_{t o l}{ }^{2}>2 b_{n}{ }^{2} \sigma_{y d}{ }^{2}+2 a_{n}{ }^{2} \sigma_{x d}{ }^{2}+4 b_{n}{ }^{2} \sigma_{y s}{ }^{2}
\end{aligned}
$$

The same relation between the misalignment of the corrector and the misalignment of the dipole as before, $\mathrm{dx}_{\mathrm{s}}+\alpha^{*} \mathrm{dx}_{\mathrm{d}}$ and $\mathrm{dy}_{\mathrm{s}}+\beta^{*} \mathrm{dy}_{\mathrm{d}}$

$$
\begin{aligned}
& 0<2 b_{n}{ }^{2}{\sigma_{x d}}^{2}\left(1+2 \alpha^{2}\right)+2 a_{n}{ }^{2} \sigma_{y d}{ }^{2}<b_{t o l}{ }^{2} \\
& 0<2 b_{n}{ }^{2} \sigma_{y d}{ }^{2}\left(1+2 \beta^{2}\right)+2 a_{n}{ }^{2}{\sigma_{x d}}^{2}<a_{t o l}{ }^{2}
\end{aligned}
$$

this gives

$$
\begin{aligned}
& \sigma_{x d}<\sqrt{\max \left[\frac{b_{t o l}{ }^{2} b_{n}{ }^{2}\left(1+2 \beta^{2}\right)}{D}, \frac{a_{n}{ }^{2} a_{t o l}{ }^{2}}{D}\right]} \\
& \sigma_{y d}<\sqrt{\max \left[\frac{a_{t o l}{ }^{2} b_{n}{ }^{2}\left(1+2 \alpha^{2}\right)}{D}, \frac{a_{n}{ }^{2} b_{t o l}{ }^{2}}{D}\right]} \\
& \text { with } \quad D=2 b_{n}{ }^{4}\left(1+2 \beta^{2}\right)\left(1+2 \alpha^{2}\right)-a_{n}{ }^{4}
\end{aligned}
$$

For the case all dipoles have a spool piece the factor 2 goes away in front of $\alpha$ and $\beta$.

\section{ESTABLISHING ALIGNMENT TOLERANCES FROM "BUDGET"}

The errors have systematic, systematic per arc (uncertainty) and random error components. Only systematic errors combine to contribute to the systematic alignment budget. For the random budget we use the following value of the components causing feed-down

$$
\begin{aligned}
& b_{n}=\sqrt{b_{n, s y s}^{2}+b_{n, u n c}^{2}+b_{n, \text { ran }}^{2}} \\
& a_{n}=\sqrt{a_{n, \text { sys }}^{2}+a_{n, u n c}^{2}+a_{n, \text { ran }}^{2}}
\end{aligned}
$$


Table 1: Summary of feed down budget

\begin{tabular}{|c|c|c|c|c|}
\hline $\mathrm{n}$ & 2 & 3 & 4 & 5 \\
\hline Feed down effect & dipole: closed orbit & quadrupole: $\beta$ beat & $\begin{array}{c}\text { sextupole: } \\
\text { resonances, Q' }\end{array}$ & $\begin{array}{c}\text { octtupole: } \\
\text { anharmonicities Q', }\end{array}$ \\
\hline $\begin{array}{c}\text { Tolerable systematic } \\
\mathrm{a}_{\mathrm{n}-1}\end{array}$ & $10 \%$ of dipole $(0.5 \mathrm{u})$ & $1 / \sqrt{8}$ unc. $(0.3 \mathrm{u})$ & $1 / \sqrt{8}$ unc. $(0.3 \mathrm{u})$ & $1 / \sqrt{8}$ unc. $(0.19 \mathrm{u})$ \\
\hline $\begin{array}{c}\text { Tolerable systematic } \\
\mathrm{b}_{\mathrm{n}-1}\end{array}$ & $10 \%$ of dipole $(0.5 \mathrm{u})$ & $1 / \sqrt{8}$ unc. $(0.3 \mathrm{u})$ & $1 \mathrm{u}$ & $\left.0.07 \mathrm{u}(\mathrm{Q})^{\prime \prime}\right)$ \\
\hline $\begin{array}{c}\text { Tolerable random } \\
\mathrm{a}_{\mathrm{n}-1}\end{array}$ & $\begin{array}{c}14 \% \text { of random in } \\
\text { dipole }(1.0 \mathrm{u})\end{array}$ & $\begin{array}{c}10 \% \text { of coupling } \\
(0.8 \mathrm{u})\end{array}$ & $\begin{array}{c}10 \% \text { of increase } \\
=46 \% \text { of total }(0.2 \mathrm{u})\end{array}$ & $\begin{array}{c}10 \% \text { of increase } \\
=46 \% \text { of total }(0.3 \mathrm{u})\end{array}$ \\
\hline $\begin{array}{c}\text { Tolerable random } \\
\mathrm{b}_{\mathrm{n}-1}\end{array}$ & $\begin{array}{c}14 \% \text { of random in } \\
\text { dipole }(1.0 \mathrm{u})\end{array}$ & $7 \% \beta$ beat $(0.36 \mathrm{u})$ & $\begin{array}{c}10 \% \text { of increase } \\
=46 \% \text { of total }(0.7 \mathrm{u})\end{array}$ & $\begin{array}{c}10 \% \text { of increase } \\
=46 \% \text { of total }(0.3 \mathrm{u})\end{array}$ \\
\hline
\end{tabular}

For each multipole we set up a budget for the feeddown harmonic $\mathrm{n}-1$ for systematic, uncertainty and random errors separately, for both random and skew components. From this budget we calculate systematic, and random parts of the tolerated alignment. It is checked that second-order ( $\mathrm{n}-2)$ contributions can be ignored.

The table of field imperfections used is the "9901" table. It gives values of the field components expressed in units (u) of $10^{-4}$ relative to the main field.

Table 1 gives the summary of the budget for calculation of the tolerances. For $\mathrm{n}=2$ the feed down component is only a dipolar effect. Random $b_{1}$ and $a_{1}$ allowed is $14 \%$ of random $b_{1}$ and $a_{1}$ in the field table, which corresponds to $1 \%$ of aperture budget, i.e. noise.

For the calculation of the systematic feed-down from $a_{3} / b_{3}$ we do not take the value for the $a_{3}$ and $b_{3}$, which are too small, from the error table but the value is divided by $\sqrt{8}$ (random distribution over the 8 arcs) which gives 0.3 . For the $\mathrm{a}_{2} / \mathrm{b}_{2}$ budget (feed down from $\mathrm{a}_{3} / \mathrm{b}_{3}$ ) a tune shift or coupling coefficient of 0.03 has to be accommodated. A $\beta$-beating of $7 \%$ gives us a random $b_{2}$ of 0.36 . As random $\mathrm{a}_{2}$, an increase of the random component of $10 \%$ (allowed by the correction system) is taken which gives $46 \%$ of the $\mathrm{a}_{2}$ in the field table.

For the systematic $a_{4} / b_{4}$ feed-down the values in the field table is divided by $\sqrt{8}$ (same argument as for $a_{3} / b_{3}$ ). The systematic and uncertainty for the feed-down budget are taken as $10 \%$ of the $a_{3} / b_{3}$ components in the field table and the random $a_{3} / b_{3}$ are taken as $46 \%$ of the random components (increases the random component by $10 \%$ ).

For the $a_{5} / b_{5}$ feed down we take the values of $a_{5} / b_{5}$ from the table. The Q" budget requires the systematic $\mathrm{b}_{4}$ to be less than 0.1. If we assume that the systematic and the uncertainty errors of the field contribute equally

$$
\sqrt{b_{4 s}{ }^{2}+\left\langle b_{4 u}\right\rangle^{2}}=0.1 \mathrm{u}
$$

then the systematic $b_{4}$ is $0.1 / \sqrt{2}=0.07 \mathrm{u}$. The systematic $a_{4}$ is set to the rms of the acceptable uncertainty which gives $0.55 / \sqrt{8}=0.19$. The $b_{4}$ uncertainty is taken as $0.1 / \sqrt{2} \cdot \sqrt{8}=0.2$ u. The $a_{4}$ uncertainty is defined by the dynamic aperture and is $0.55 \mathrm{u}$.

The random $b_{4} / a_{4}$ is taken as $46 \%$ of the random component of $b_{4}$, i.e. it increases the random component by $10 \%$.
For $\mathrm{a}_{6} / \mathrm{b}_{6}$ and $\mathrm{a}_{7} / \mathrm{b}_{7}$ feed down the budget is supposed to be $10 \%$ of the allowed component in the error table except for the $a_{5}$ and $a_{6}$ budget where the value is supposed to be of the same order as in the error table.

\section{SUMMING UP RESULTS}

Using the formulas above with the established budget, taking the most constraining misalignment for the dipole itself and for the $b_{3}, b_{4}$, and $b_{5}$ correctors separately, gives the result shown in Table 2. For the dipole itself the misalignment in the $\mathrm{x}$-direction is limited by the contribution from the $b_{3}$ multipole feed down and for the $y$-direction the $b_{3}$ and the $b_{5}$ are the most constraining. The misalignments of each spool piece corrector are taken relative to the dipole average axis.

\begin{tabular}{|lll|}
\hline & SUMMARY & \\
& Systematic & Random (rms) \\
$\mathrm{dx}$, dipole $\left(\mathrm{b}_{3}\right)$ & 0.14 & 0.29 \\
$\mathrm{dy}$, dipole $\left(\mathrm{b}_{3}\right)$ & 0.14 & 0.93 \\
dy, dipole $\left(\mathrm{b}_{5}\right)$ & 1.6 & 0.42 \\
$\mathrm{dx}, \mathrm{b}_{3}$-corrector & 0.09 & 0.18 \\
$\mathrm{dy}, \mathrm{b}_{3}$-corrector & 0.09 & 0.61 \\
$\mathrm{dx}, \mathrm{b}_{4}$-corrector & 31.9 & 1.9 \\
dy, $\mathrm{b}_{4}$-corrector & 20.1 & 1.6 \\
$\mathrm{dx}, \mathrm{b}_{5}$-corrector & 0.41 & 0.35 \\
$\mathrm{dy}, \mathrm{b}_{5}$-corrector & 1.07 & 0.28 \\
\hline
\end{tabular}

Table 2: Summary of the alignment tolerances in mm for the dipole (w.r.t. reference c.o.) and the correctors (w.r.t. the dipole axis.

The random alignment tolerance of the $b_{3}$ spool piece of $0.18 \mathrm{~mm}$ is probably not achievable. A complete aperture balance has to be remade (the $\beta$ beating budget has to be re-evaluated). The random tolerance of $0.29 \mathrm{~mm}$ for the dipole is smaller than the r.m.s. closed orbit distribution and this would also imply a revision of the aperture budget. For the $b_{5}$ corrector the tolerance is pessimistic as the effect of random $a_{4} / b_{4}$ on dynamic aperture is probably negligible (should be evaluated).

\section{REFERENCES}

[1] LHC Alignment Workshop, CERN Dec. 2000, http://wwwslap.cern.ch/ vrd/aplaw/page.html 